\title{
Plasmaspheric Electron Content contribution inferred from ground and radio occultation derived Total Electron Content
}

\author{
Angela Aragon Angel, Jaume Sanz, Jose Miguel Juan, Manuel Hernandez Pajares \\ Research group of Astronomy and Geomatics \\ Technical University of Catalonia \\ Barcelona, Spain \\ angela@ma4.upc.edu
}

David Altadill

Observatory del Ebro

Universitat Ramon LLull

Roquetes, Spain

\begin{abstract}
The FORMOSAT-3/COSMIC satellite constellation has become an important source of remote sensing data globally distributed for the sounding of the atmosphere of the Earth and, in particular, the ionosphere. In this study, electron density profiles derived using the Improved Abel transform inversion in Radio Occultation (RO) scenarios are used as input data to derive some features regarding the topside and outside ionospheric contribution, hence, the plasmasphere in great extend, by means of the analysis of the integral values of the shape functions corresponding to each density profile. The novelty presented in this paper, with respect to previous works, is the use of experimental data from the FORMOSAT-3/COSMIC RO-derived electron densities to infer global characterizations and distribution of the Total Electron Content (TEC) into its main components: ionospheric TEC and plasmaspheric TEC, both contributing to TEC. The results show agreement with earlier modeled and observational data from previous local studies of the plasmaspheric contribution. The main conclusion of this research is that the plasmasphere contributes significantly to TEC and the ratio between plasmaspheric TEC and TEC has been climatologically analyzed for the whole year 2007 confirming that the major relative impact of the plasmasphere is during night time. The added value of this study is that the results obtained are globally distributed and can help to infer a proxy for the plasmaspheric contribution at any location over the globe thanks to the FORMOSAT-3/COSMIC coverage.
\end{abstract}

Keywords-Ionosphere; Plasmasphere; GPS; radio occultation; electron density; TEC; Abel transform; Separability; FORMOSAT-3/COSMIC

\section{INTRODUCTION}

In the past years, many efforts have been devoted to try and successfully model the topside contribution of the ionosphere. Most of such efforts rely on the availability of global measured data provided either by direct measurements from satellitebased ionosondes on board satellite missions such as ISIS (International Satellite for Ionosphere Studies)-1, ISIS-2 [3] and IK (Intercosmos)-19 (information about this mission is to be found at http://www.astronautix.com/craft/auos.htm) or predictions based on bottom-side ionospheric measurements from ground-based ionosondes [10]. According to literature [3] [7], estimates of the contribution of the Plasmaspheric Electron Content (PEC) may vary from 10\% of the Total Electron Content (TEC) during daytime hours, when the density of the F2 region is highest, to approximately $50 \%$ during night time, when the F2 region density is lowest. More recently, models based on both, TEC derived from ground receivers of signals of the Global Positioning System satellites (GPS) and ionosonde data, have proven to be able to locally reconstruct the topside ionospheric electron density contribution (e.g. [12]; [8]). The current study is focused on providing a global description for the plasmaspheric contribution based on information retrieved from electron densities derived from FORMOSAT-3/COSMIC (F-3/C) Radio Occultation (RO) data. The dense coverage of these satellite mission RO events in longitude, latitude and local time makes this constellation become a powerful tool to retrieve ionospheric and tropospheric information all time, all weather. So far, this constellation has been used to monitor the ionosphere and the troposphere, but, as it will be discussed, the interrelationship between the electron density profiles derived from ionospheric RO (IRO) and TEC will provide a way to also monitor the plasmaspheric content within the TEC. A simple model for the plasmaspheric portion within the TEC has also been implemented and able to reproduce the upper contribution of EC better.

\section{ELECTRON DENSITY: SEPARABILITY, EXTRAPOLATION SCHEME AND PROFILE NORMALIZATION}

This study is based on electron densities retrieved from F3/C RO data by means of the Improved Abel transform. This inversion method is based on the separability hypothesis, firstly introduced in [9]. Separability consists of the following: 
the electron density $N e(\lambda, \varphi, \mathrm{h})$ can be expressed by means of a function $F$ that assumes the height dependency, called shape function, and the Total Electron Content (TEC) associated to the corresponding horizontal coordinates $(\lambda, \varphi)$, as reflected in the next equation:

$$
\operatorname{Ne}(\lambda, \varphi, \mathrm{h})=\operatorname{VTEC}(\lambda, \varphi) \cdot \mathrm{F}(\mathrm{h})
$$

With the aid of externally provided TEC values (for instance, Vertical TEC -VTEC-derived from GPS ground data) at each corresponding location $(\lambda, \varphi)$, the new unknown to be solved is not $N e$ but $F(h)$. Thanks to the inclusion of $T E C$ information, separability helps overcoming the limitations of the spherical symmetry assumption when applying the classical Abel transform. Several works have proven that the use of the separability concept in the inversion procedure leads to better determinations of ionospheric electron densities regardless of the observable used as main input: either the combination of GPS carrier phases L1 and L2 [6] or the bending angle of L1 (improvements up to $45 \%$ shown in [1]).

In the inversion process, the contribution of the electron content above the Low Earth Orbit (LEO) is, in principle, one important matter that has to be taken into account when inverting RO measurements. This upper electron contribution should be accounted for in a simple but reasonable way in order to further improve the inversion procedure. Based on the properties of an extrapolation scheme to describe the upper behavior of real $N e$ profiles and taken into account that the profile only reaches up to the LEO height, one approach to the problem would be to perform a modified exponential extrapolation, which includes a bias, of the density profile $N e$. The exponential extrapolation is often used for representing the topside part of the electron density profiles (e.g. [8]).

In [1], several extrapolation schemes were tested to find out that the relative error of the profiles when compared to calibrated ionosonde data only improved about $2 \%$. For LEOs at higher orbital heights, all the extrapolations are compensated with the values of $F(h)$ for high values of $h$ as can be seen in Figure 1. $F(h)$ basically accounts for the ionosphere (no plasmasphere) as can be derived from the results. This concludes that, regardless of the plasmaspheric model used, its contribution to IRO is minimal i.e. the derived IRO profile is quite insensitive to the electron density above the LEO.

Another way to confirm the former conclusion is by integrating the shape function $F(h)$. Indeed, extrapolating $F(h)$ above the LEO orbit, one can integrate Eq. (1) for any given set of fixed coordinates $\left(\lambda_{0}, \varphi_{0}\right)$ :

$$
\int_{0}^{\infty} N_{e} d h=\operatorname{VTEC}\left(\lambda_{0}, \varphi_{0}\right) \int_{0}^{\infty} F(h) d h
$$

The left-hand part of (2) corresponds to the TEC of the RO profile $\left(\mathrm{TEC}_{\mathrm{IRO}}\right)$ while the integral on the right-hand part is the integral of the shape function $(I S F)$. If the $\mathrm{TEC}_{\mathrm{IRO}}$ were all vertical VTEC, ISF should be 1 . Therefore, $I S F$ accounts for the ratio between the $\mathrm{TEC}_{\mathrm{IRO}}$ and the VTEC.

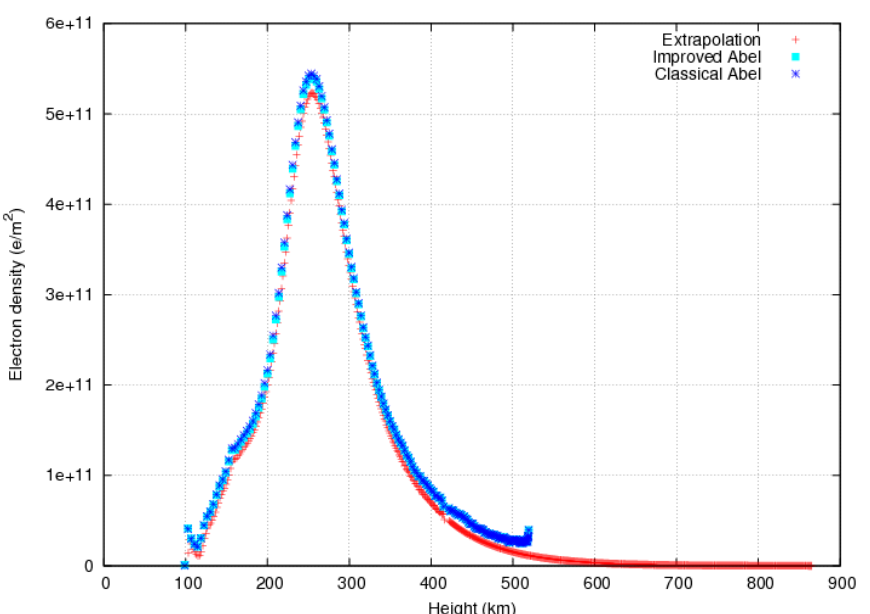

Figure 1: Example of extrapolated profile vs. original profile derived using classical and improved Abel inversion for February 24, 2007, involving FORMOSAT-3/COSMIC 3 and PRN10 at 12h 54min UT.

However, in practice, after reviewing more than 126,000 shape functions corresponding to RO-derived electron density profiles using the separability hypothesis for the year 2007, it turned out that this was not the case for all shape functions deduced from real data: Figure 2 represents the histogram of ISF derived from processing Level 0 products from the COSMIC Data Analysis and Archive Center (CDAAC) corresponding to fifty-three days in 2007 evenly distributed throughout the year (one day per week). Figure 2 clearly shows that part of the ISF values presented here are typically far below from the hypothetical value of one. This would mean that the exponential extrapolation of the $F(h)$ typically underestimates the fraction of the electron content above the RO measured profile. Therefore, it was needed to try and understand this unexpected behavior of the integral value of the shape functions. For this reason, dependencies of the distribution of these ISF values with respect latitude, local time, and season were analyzed while seeking for an explanation to the non fulfillment of (2). Regarding latitude, results did not associate the lowest integral values to any particular latitudinal band considered. On the contrary, the two-modal distribution of the integral values appears for all latitudes. Regarding local time, ISF values were classified into night time and day time when considering the local time (LT) of occurrence of the associated RO event (see Figure 4): daytime from 10 to $16 \mathrm{LT}$ and night time from 22 to $04 \mathrm{LT}$. These results revealed that there is a marked dependence of the $I S F$ on local time. Figure 3 is also able to explain the two modal distributions noticed in Figure 2. It is during daytime when the higher integral values for the corresponding shape functions are found whereas during nighttime, the lower ones are found. In other words, the $\mathrm{TEC}_{\mathrm{IRO}}$ is typically lower than the $50 \%$ during nighttime and around $70 \%$ during day time.

Summing up, the principal conclusion is that there is an under-determination of the integral values of the shape functions as evidenced in Figure 2. The under-determination of the integral values of the shape functions reveals certain inconsistencies between the RO-associated electron content

This work has been partially supported by the Spanish Ministry of Science and Innovation under the CTM2010-21312-C03-02 project. 
and the VTEC that are related with the protonospheric contribution to the GPS-derived VTEC, contribution that is basically missing in the RO-derived data.

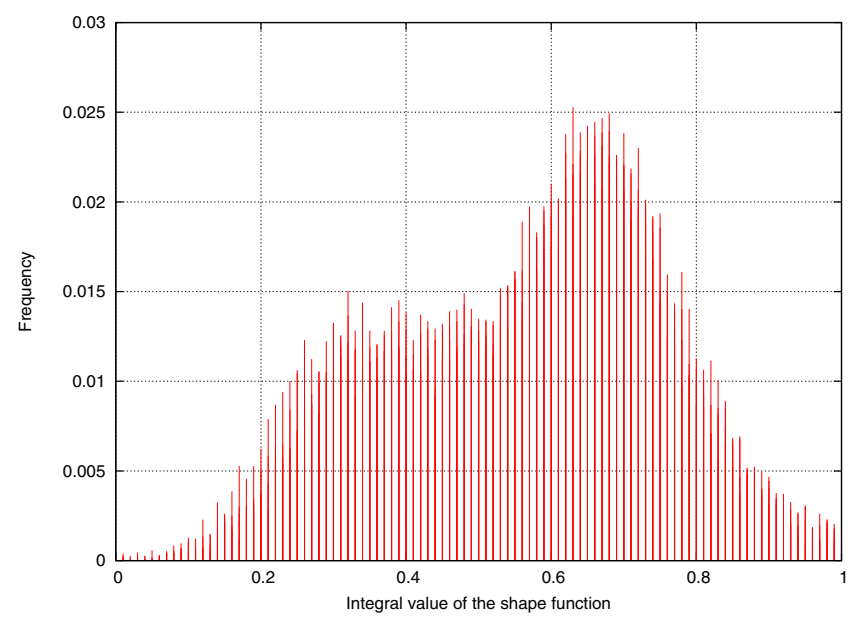

Figure 2: Histogram of integral values of shape functions corresponding to RO derived electron densities using the separability hypothesis. Note the two modal distributions of such values around 0.32 and 0.6.

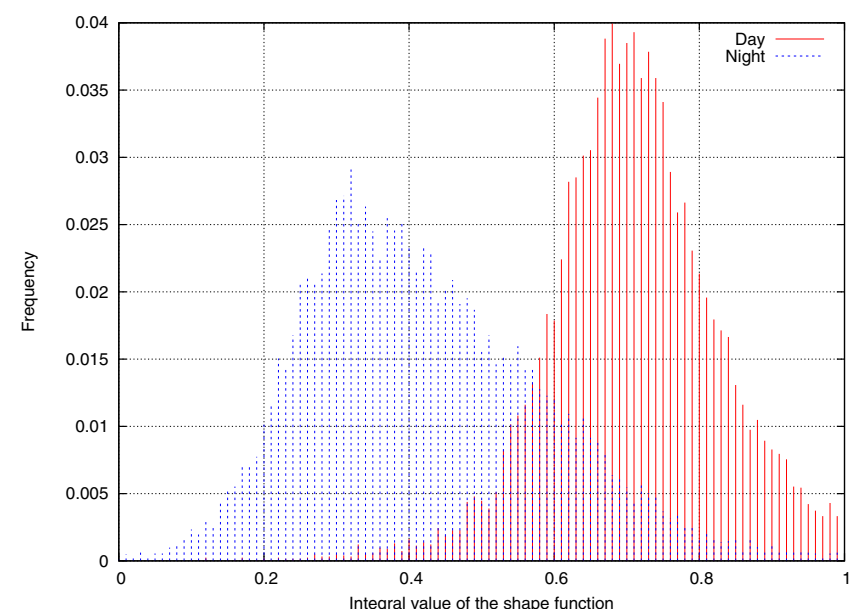

Figure 3: Histogram of integral values of shape functions corresponding to Figure 2 discriminating local nigh vs. local day, evidencing a clear diurnal dependence.

It is well known that, at a given altitude $\mathrm{h}$ in the topside ionosphere, the electron density $\mathrm{Ne}$ can be considered as a sum of the constituent major ions $\mathrm{O}^{+}$and $\mathrm{H}^{+}$densities, $\mathrm{NO}^{+}$and $\mathrm{NH}^{+}$respectively,

$$
N_{e}(h)=N_{O}^{+}(h)+N_{H}^{+}(h)
$$

However, at above some level in the topside ionosphere, the lighter ions $\left(\mathrm{H}^{+}\right)$turn to dominate (starting the protonosphere) while the heavier ions $\left(\mathrm{O}^{+}\right)$do dominate below such level of the topside ionosphere. Such level is called transition level or transition height [5] which changes as function of solar activity, season, latitude and it locates between 500 and over $1000 \mathrm{~km}$ for low solar activity [11]. The way we obtain the shape functions to extrapolate the RO profiles is very similar to the way described by [11] for obtaining the so called vertical scale height (VSH). The parameter $h_{T}$ that we use means a good estimate of the topside
VSH and $F(h)$ should account for the contribution to the topside $N_{e}(h)$ coming from the vertical distribution of $\mathrm{O}^{+}$only and that coming from $\mathrm{H}^{+}$has been missed out. Therefore, the integral values of $F(h), I S F$, (2) should not account for the protonospheric contribution to the GPS-VTEC, avoiding in this way the underestimation of ISF from its hypothetical value of 1. In order to justify this affirmation, in Figure 4, it is depicted the relationship of the integral shape function $(I S F)$ and the ratio of the lower layer TEC (TEC1) with respect the total TEC $(T E C 1+T E C 2)$ corresponding to the sum of $T E C$ of the first (TEC1) and second layer (TEC2) into which the ionosphere has been divided, obtained from a tomographic approach in the framework of the European Space Agency project Precise Real Time Orbit Determination and Clock Synchronization (PRTODTS). The data correspond to November $24^{\text {th }} 2009$; the $I S F$ values associated to RO occurring at latitudes between $40^{\circ}$ and $60^{\circ}$ for all longitudes are depicted versus the fraction of $T E C 1 /(T E C 1+T E C 2)$ as functions of the local time (LT) expressed in seconds.

As one can see, there is a reasonable agreement between the $I S F$ values and the ratio of the TEC associated to the first layer when compared to the total TEC, basically meaning that the $I S F$ accounts for such ratio, i.e. the ionospheric component of the total TEC. Moreover, in an indirect way and interpreting the information in this plot in terms of the two-layer tomographic approach of the ionosphere, TEC2 would basically account for the topside ionospheric contribution, that could be added to the RO TEC (mainly, bottomside ionosphere) to account for the total TEC. This fact would open the possibility to provide topside maps from the 2-layer tomographic approach of the ionosphere.

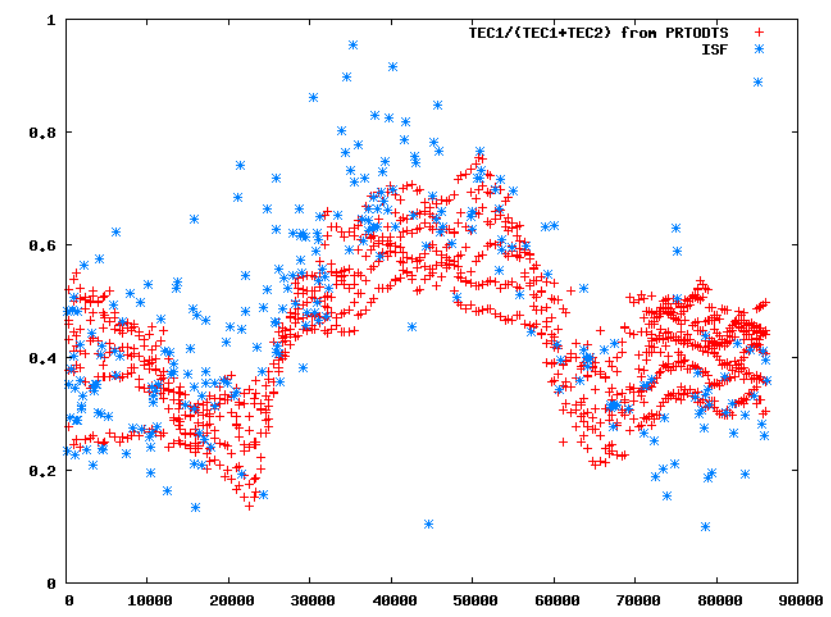

Figure 4: For RO occurring in latitudes between $40^{\circ}$ and $60^{\circ}$ during November 24th 2009, comparison of the ISF for all longitudes (blue stars) obtained from FORMOSAT-3/COSMIC radio occultation GPS data and the fraction of TEC corresponding to the lower layer for European longitudes from ground GPS data (source: PRTODTS, red crosses). 


\section{IONOSPHERIC VERSUS PLASMASPHERIC CONTRIBUTION TO TOTAL TEC}

In [7], a preliminary study of the plasmasphere fraction of the TEC was presented. The analysed parameter was the socalled "plasmaspheric fraction" i.e. the ratio of the plasmaspheric electron content $(E C p l)$ to the ionospheric $T E C$ (ECion):

$$
r=\frac{E C_{p l}}{E C_{i o n}}
$$

In that work, $E C p l$ was estimated integrating ISIS-1, ISIS-2 and IK-19 electron density profiles at altitudes from $1000 \mathrm{~km}$ to the plasmapause using SMI-86 plasmaspheric density model [4], and ECion corresponding to ionospheric TEC observed and modelled below $1000 \mathrm{~km}$ (they actually included bottomside $E C-1$ produced with IRI adjusted to observations of the F2-layer peak by ionosondes, and $E C$-2 calculated from observed topside $N_{e}(h)$ profiles or simulated with IRI in order to obtain $E C i o n=E C-1+E C-2)$. From the study in [7], they concluded that the plasmaspheric fraction comprised from 20 up to $50 \%$ of the ionospheric electron content in most cases.

The main goal of the current study was to infer what the plasmaspheric contribution would be when using RO derived TEC (obtained from real direct measurements) since the majority of the results trying to account for the plasmaspheric contribution to total $T E C$ relied on simulated or partially simulated data.

Firstly, the "plasmaspheric fraction" $r$ has been reinterpreted in terms of the value of the integral of the shape function (ISF) leading to the following expression:

$$
r=\frac{1}{I S F}-1
$$

Starting from the experiment data set described in Section II, it has been pursued to try and find out whether the same relationships as in [7] apply or not when using actual real data, not modelled ones. Figure 5 and Figure 6 show the reinterpretation of the information presented in Figure 2 and Figure 3 by means of using (5).

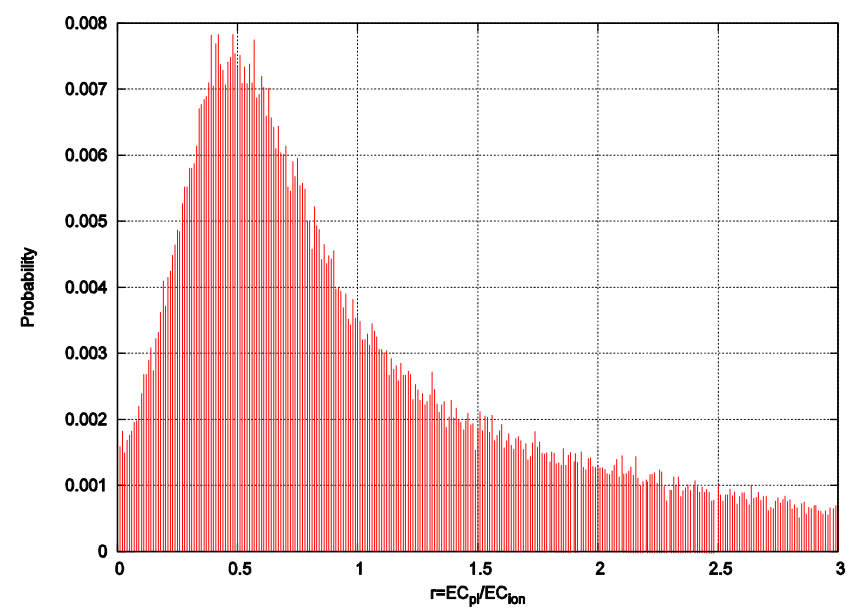

Figure 5: Probability of the plasmaspheric fraction $\mathrm{r}$ corresponding to RO derived electron densities using the separability hypothesis used to depict Figure 2.

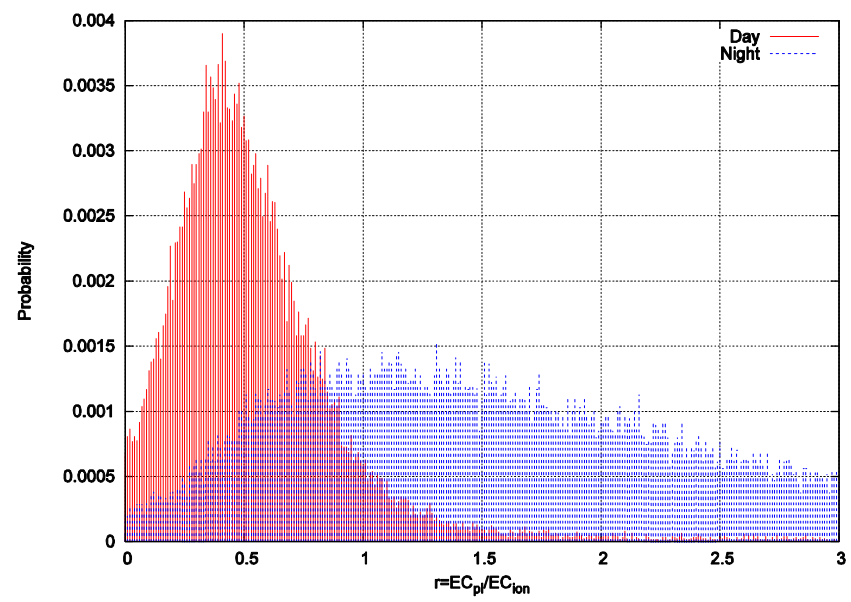

Figure 6: Probability of $r$ classified with respect local time; reinterpretation of Figure 3 in terms of $r$.

Results in Figure 5 and Figure 6 are in agreement with those presented in [7] in spite of the different data nature (partially real + modelled in [7] versus real in the current work). These results also reveal that there is a marked dependence of the plasmaspheric fraction on local time, as expected from results in Figure 3. It is during daytime that the highest values for such ratio are found.

Thirty days corresponding to winter (day of year 1 to 30), spring (day of year 90 to 120), summer (day of year 180 to 210 ) and autumn (day of year 270 to 300 ) respectively have been compared to try to evidence some seasonal dependence with respect the distribution of $r$. In Figure 7 and Figure 8, results for day time and night time are respectively shown for the above selected periods. 


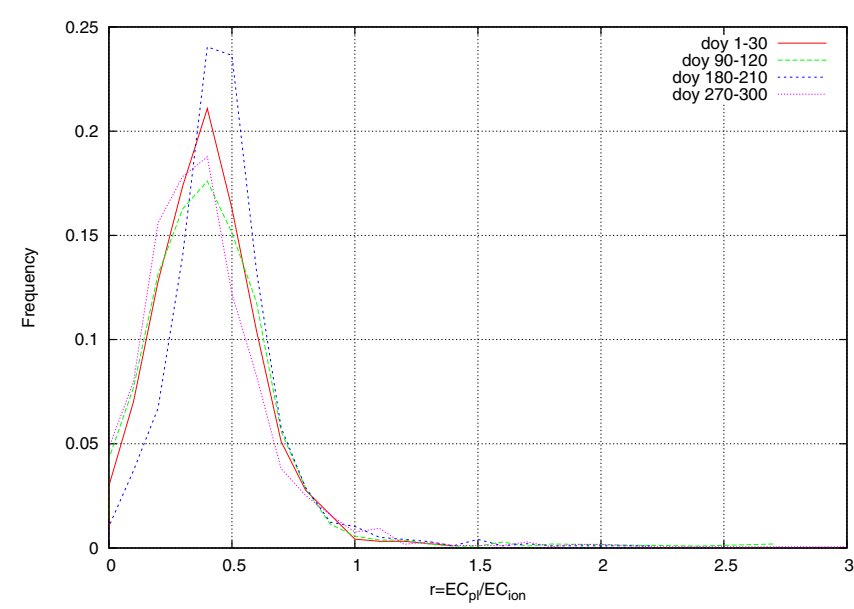

Figure 7: Seasonal dependence with respect the distribution of $r$ for days corresponding to winter (doy 1 to 30 ), spring (doy 90 to 120), summer (doy 180 to 210 ) and autumn (doy 270 to 300 ) for day time.

On one hand, for both solstices (red and blue lines in Figure 7 ), there is a more pronounced peak of occurrences around $r=0.41$ during day time, whereas for both equinoxes (green and pink lines in Figure 7) the distribution of the parameter $r$ presents a tail of $r$ values ranging from $r=2$ almost up to $r=3$, where no occurrence of $r$ values is found for solstices. On the other hand, no clear different behavior is found between the four epochs of the year while looking at Figure 8.

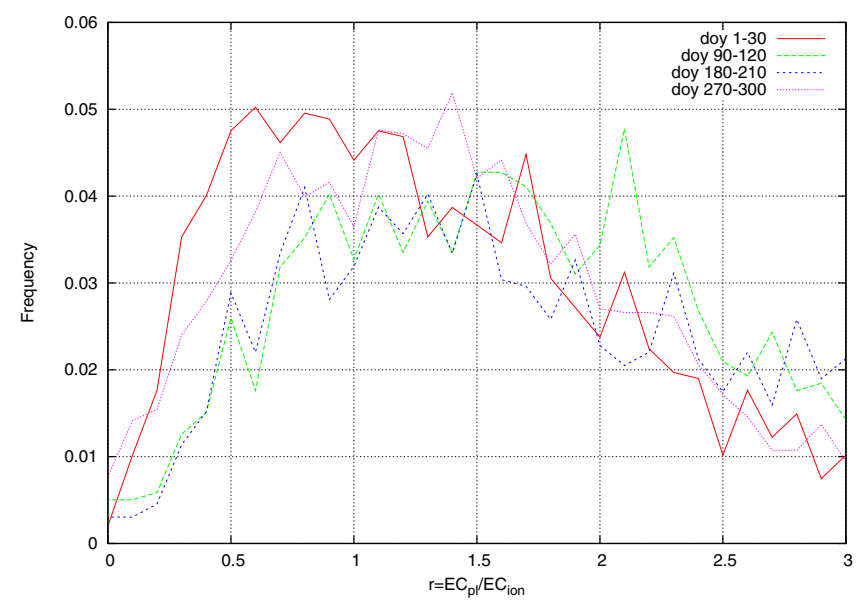

Figure 8: Seasonal dependence with respect the distribution of $\mathrm{r}$ for days corresponding to winter (doy 1 to 30), spring (doy 90 to 120), summer (doy 180 to 210 ) and autumn (doy 270 to 300 ) for night time.

\section{FEASIBILITY TO MODEL THE PLASMASPHERIC CONTRIBUTION}

As a consequence of the results shown and discussed in previous sections, the value of ISF mainly provides information about the ionospheric content. This implies that the value $1-I S F$ informs about the associated plasmaspheric contribution. Therefore, by multiplying these quantities for the corresponding VTEC, the value $V T E C \cdot I S F$ would account for the ionospheric VTEC $\left(\right.$ TEC $\left._{\text {ion }}\right)$ and VTEC. $(1-I S F)$ for the plasmaspheric TEC $\left(\mathrm{TEC}_{\mathrm{pl}}\right)$. Thus, by modeling the ISF behavior, it would be possible to infer the plasmaspheric contribution, allowing correcting current VTEC maps by accounting for this upper term. This is a matter that requires further efforts in a devoted study.

\section{CONCLUSIONS}

The novelty presented in this paper with respect to previous works is the use of experimental data from the FORMOSAT3/COSMIC RO-derived electron densities to infer global characterizations and distribution of the Total Electron Content (TEC) into its main components: ionospheric TEC and plasmaspheric TEC, both contributing to total TEC. The main conclusion from this preliminary assessment of the plasmaspheric electron content contribution inferred from RO data is that, by working solely with electron densities derived from RO scenarios, the contribution of the protonosphere into the total electron content is systematically being neglected, mostly accounting for the ionospheric contribution. This contribution can reach from 20 up to $50 \%$. By considering an effective height for each contribution (protonospheric + ionospheric) by means of a two layer ionospheric model, an ideal solution for ground-based GNSS applications would be obtained, one layer to model the protonosphere and another for the ionosphere. It has been shown that by modeling in such way, the results that were obtained with RO data analysis can be validated. The results are in agreement with earlier modeled and observational data from previous local studies of the plasmaspheric contribution.

\section{ACKNOWLEDGMENT}

The authors would like to thank the University Corporation for Atmospheric Research (UCAR), the National Space Organization (NSPO) in Taiwan for the availability of FORMOSAT-3/COSMIC constellation raw data.

\section{REFERENCES}

[1] A. Aragon - Angel, Y. - A. Liou, C. - C. Lee, B. W. Reinisch, M. Hernández - Pajares, M. Juan, and J. Sanz, "Improvement of retrieved FORMOSAT - 3/COSMIC electron densities validated by ionospheric sounder measurements at Jicamarca," Radio Sci., 46, 2011, doi:10.1029/2010RS004578

[2] A. Aragon-Angel, M. Hernandez-Pajares, J.M. Juan, J. Sanz, "Improving the Abel transform inversion using bending angles from FORMOSAT-3/COSMIC," GPS Solut, 2009, Springer-Verlag

[3] R.F. Benson and D. Bilitza, "New satellite mission with old data: Rescuing a unique data set," Radio Sci., 44, 2009, RS0A04, doi:10.1029/2008RS004036

[4] Yu. K. Chasovitin, "Earth's ionosphere and plasmasphere model," 1999, http://zeus.wdcb.ru/wdcb/stp/mod_I_P

[5] K. Davies, "Ionospheric Radio," Chapter 5, IEE Electromagnetic Waves Series 31, Peter Peregrinus Ltd , 1990, ISBN 086341186 X, pp. 124154

[6] M. Garcia-Fernandez, "Contributions to the 3D ionospheric sounding with GPS data," Doctoral Thesis, Technical University of Catalonia (UPC), 2004, B.45104-2004/84-688-8156-2

[7] T. L. Gulyaeva, X. Huang, B.W. Reinisch, "Plasmaspheric extension of topside electron density profiles," Avd. Space Res. Vol. 29, No. 6, 2002, pp. $825-831$

[8] Hargreaves, J. K., "The solar-terrestrial environment”, Cambridge University Press, 1992, Cambridge UK. 
[9] M. Hernandez-Pajares, J. M. Juan, J. Sanz, "Improving the Abel inversion by adding ground data LEO radio occultations in the ionospheric sounding," Geophys Res Lett 27, 2000, pp. 2743-2746

[10] X. Huang, B.W. Reinisch, "Vertical electron density profiles from the Digisonde network,” Adv. Space Res., Vol. 18, 2001, pp. 21-29

[11] I. Kutiev, P. Marinov, "Topside sounder model of scale height and transition height characteristics of the ionosphere" Adv. Space Res. 39, 2007, 759-766, doi:10.1016/j.asr.2006.06.013
[12] P. Sibanda, L.A. McKinnell, "Topside ionospheric vertical electron density profile reconstruction using GPS and ionosonde data: possibilities for South Africa," Ann. Geophys., Vol. 29, 2011, pp. 229236

[13] S. M. Stankov, Ko. Stegen, P. Muhtarov and R. Warnant, "Local ionospheric electron density profile reconstruction in real time from simultaneous ground-based GNSS and ionosonde measurements" Adv. Space Res. 47, 2011, 1172-1180, doi:10.1016/j.asr.2010.11.039. 